\title{
Erratum to: Up-converted fluorescence from photosynthetic light- harvesting complexes linearly dependent on excitation intensity
}

\author{
Kristjan Leiger $^{1} \cdot$ Arvi Freiberg $^{1,2}$
}

Published online: 2 April 2015

(C) Springer Science+Business Media Dordrecht 2015

\section{Erratum to: Photosynth Res \\ DOI 10.1007/s11120-015-0117-x}

The last sentence in the Acknowledgements section in the original publication was incorrect. It should have read as follows:

The authors thank C. N. Hunter, J. D. Olsen, A. A. Moskalenko, and A. A. Ashikhmin for kindly providing the samples, and I. I. Proskuryakov for useful discussions.

The online version of the original article can be found under doi:10.1007/s11120-015-0117-x.

Kristjan Leiger

Kristjan.Leiger@ut.ee

Arvi Freiberg

arvi.freiberg@ut.ee

1 Institute of Physics, University of Tartu, Ravila 14c,

51011 Tartu, Estonia

2 Institute of Molecular and Cell Biology, University of Tartu,

Riia 23, 51014 Tartu, Estonia 